\title{
On the Long-Time Behavior of Ferroelectric Systems
}

\author{
J. M. Greenberg* $\dagger$ \\ R. C. MacCamy ${ }^{\dagger}$ \\ C. V. Coffman ${ }^{\dagger}$
}

\section{Introduction}

\section{A. Summary of Results.}

In this note we investigate a new model for the behavior of ferroelectric materials. This model is analogous to one used in [1] to describe the dynamics of elastic materials which exhibit phase changes.

We begin with Maxwell's equations which we take in the form,

$$
\frac{\partial \mathbf{B}}{\partial t}=-\operatorname{curl} \mathbf{E} \text { and } \frac{\partial \mathbf{D}}{\partial t}+\sigma \mathbf{E}=\operatorname{curl} \mathbf{H}
$$

Here $\mathbf{E}$ and $\mathbf{H}$ are the electric and magnetic fields and $\mathbf{D}$ and $\mathbf{B}$ the electric and magnetic displacements, and $\sigma \geq 0$ is the conductivity.

In usual dielectrics, which are homogeneous and isotropic, $\mathbf{D}$ and $\mathbf{E}$ and $\mathbf{B}$ and $\mathbf{H}$ are related by the constitutive equations,

$$
\mathbf{D}=\epsilon \mathbf{E} \text { and } \mathbf{B}=\mu \mathbf{H}
$$

when $\epsilon$ and $\mu$ are positive constants. Insertion of (1.2) into (1.1) yields a linear hyperbolic system which can be solved in a region $\Omega$ subject to initial values for $\mathbf{E}$ and $\mathbf{H}$ and the specifications of the tangential components of either $\mathbf{E}$ or $\mathbf{H}$ on $\partial \Omega$. Our primary focus will be on the case where $\mathbf{n} \times \mathbf{E}=\mathbf{0}$ on $\partial \Omega$ but we will describe briefly what happens when $\mathbf{n} \times \mathbf{H}=\mathbf{0}$ on $\partial \Omega$.

In the case of ferroelectric materials one replaces (1.2) by,

$$
\mathbf{D}=\epsilon(\mathbf{E}+\mathbf{P}) \text { and } \mathbf{B}=\mu \mathbf{H}
$$

where $\mathbf{P}$ is the electric polarization.

We regard the polarization as an internal field which evolves according to its own Maxwell type system; namely

$$
\epsilon_{1}\left(\frac{\partial \mathbf{P}}{\partial t}+\frac{\mathbf{j}}{\delta}\right)=\operatorname{curl} \mathbf{m}
$$

and

*This research was partially supported by the Applied Mathematical Sciences Program, U.S. Department of Energy and the Mathematics and Computer Science Division, Army Research Office.

${ }^{\dagger}$ Carnegie Mellon University, Department of Mathematical Sciences, Pittsburgh, PA 15213. 


\section{DISCLAIMER}

This report was prepared as an account of work sponsored by an agency of the United States Government. Neither the United States Government nor any agency thereof, nor any of their employees, makes any warranty, express or implied, or assumes any legal liability or responsibility for the accuracy, completeness, or usefulness of any information, apparatus, product, or process disclosed, or represents that its use would not infringe privately owned rights. Reference herein to any specific commercial product, process, or service by trade name, trademark, manufacturer, or otherwise does not necessarily constitute or imply its endorsement, recommendation, or favoring by the United States Government or any agency thereof. The views and opinions of authors expressed herein do not necessarily state or reflect those of the United States Government or any agency thereof. 


\section{DISCLAIMER}

Portions of this document may be illegible in electronic image products. Images are produced from the best available original document. 


$$
\mu_{1}\left(\frac{\partial \mathbf{m}}{\partial t}+\frac{\alpha \mathbf{m}}{\delta}\right)=-\operatorname{curl} \mathbf{P}
$$

Here, $m$ has the interpretation of an internal magnetic field and $\mathbf{j}$ is a current density which is driven by the difference between an equilibrium electric field, $\hat{\mathbf{E}}(\mathbf{P})$, and the electric field $\mathbf{E}$; that is

$$
\frac{\partial \mathbf{j}}{\partial t}+\frac{\alpha \mathbf{j}}{\delta}=\frac{\beta}{\delta}(\hat{\mathbf{E}}(\mathbf{P})-\mathbf{E}) \text {. }
$$

This latter system for $\mathbf{P}, \mathbf{m}$, and $\mathbf{j}$ reduces to

$$
\delta^{2}\left(\mathbf{P}_{t t}+\lambda^{2} \text { curl curl } \mathbf{P}\right)+\alpha \delta \mathbf{P}_{t}=\beta(\mathbf{E}-\hat{\mathbf{E}}(\mathbf{P}))
$$

which couples the polarization $\mathbf{P}$ to the electric field $\mathbf{E}$. Here $\lambda=\frac{1}{\sqrt{\epsilon_{1} \mu_{1}}}$ is the speed of light for the internal fields. For definiteness we assume that

$$
\hat{\mathbf{E}}(\mathbf{P})=\nabla_{\mathbf{P}} \Phi(\mathbf{P} \cdot \mathbf{P})=2 \mathbf{P} \Phi^{\prime}(\mathbf{P} \cdot \mathbf{P})
$$

where $s \rightarrow \Phi(s):[0, \infty) \rightarrow(-\infty, \infty)$ is smooth and satisfies $\Phi(0)=0,-\frac{1}{2} \leq \Phi^{\prime}(0)<0$ and $\Phi^{\prime \prime}(s)>0,0 \leq s ; s=a^{2}$ is the location of the unique local minimum of $\Phi$ normalized so that $\Phi\left(a^{2}\right)<0$; and for large $s$

$$
\Phi(s) \sim k s\left|\Phi^{\prime}(0)\right|, \Phi^{\prime}(s) \sim k\left|\Phi^{\prime}(0)\right|, \text { and for } p=2 \text { and } 3, \Phi^{(p)}(s) \sim O\left(\frac{1}{s^{p-1}}\right)
$$

where $k>0$. These assumptions guarantee that

$$
\Psi(\eta) \stackrel{\text { def }}{=} \Phi\left(\eta^{2}\right)
$$

is a symmetric double-well potential with a unique local maximum at $\eta=0$, unique local minimum at $\eta= \pm a$, and quadratic growth as $|\eta| \rightarrow \infty^{1}$.

We note that (1.7) with $\hat{\mathbf{E}}(\mathbf{P})$ given by (1.8) supports hysteretic effects normally associated with bistable dynamics. Specifically, if we assume that $\mathbf{E}=\mu a \mathbf{e} \sin w t$ where $\mathbf{e} \cdot \mathbf{e}=1$ and $\mu$ is large enough and look for spatially homogeneous solutions of (1.7) of the form $\mathbf{P}=a p(t) \mathbf{e}$, then $p$ satisfies

$$
\delta^{2} p_{t t}+\alpha \delta p_{t}=\beta\left(\mu \sin w t-2 p \Phi^{\prime}\left(a^{2} p^{2}\right)\right)
$$

and plots of $e$ v.s. $p$ exhibit hysteresis loops. The results of such a simulation are shown in Figures 1 and 2. These were run for the parameter values $\alpha=\beta=\mu=1, \delta=.01$, and $\omega=\pi$ when $\Phi=\frac{\left(p^{2}-1\right)^{2}}{4}$.

In order to not complicate the analysis we consider a simple geometry. We suppose the material occupies a cylinder with generators parallel to the $z$-axis and a uniform, simply connected cross section $\Omega$. We consider only fields which are independent of $z$ and have the special form,

$$
\mathbf{E}=a e \mathbf{e}_{3}, \mathbf{P}=a p \mathbf{e}_{3}, \text { and } \mathbf{H}=b\left(h_{1} \mathbf{e}_{1}+h_{2} \mathbf{e}_{2}\right)
$$

with (1.3) holding. Here, $a^{2}$ is the location of the local minimum of $\Phi$ and $b=a \sqrt{\frac{\epsilon}{\mu}}$. In this case (1.1) and (1.2) become

\footnotetext{
${ }^{1}$ Equilibrium relations of this type may be found in Landau and Lifschitz [2], p. 84-91.
} 


$$
e_{t}+p_{t}+\sigma_{1} e=c\left(h_{2 x}-h_{1 y}\right), h_{1 t}=-c e_{y}, \text { and } h_{2 t}=c e_{x}
$$

where $\sigma_{1}=\sigma / \epsilon \geq 0$ and $c=1 / \sqrt{\epsilon \mu}$ is the speed of light, and the evolution equation (1.7) for $\mathbf{P}$ reduces to

$$
\delta^{2}\left(p_{t t}-\lambda^{2} \Delta p\right)+\alpha \delta p_{t}=\beta\left(e-2 p \Phi^{\prime}\left(a^{2} p^{2}\right)\right) .
$$

where $\alpha, \beta, \delta$, and $\lambda$ are positive constants and $\Delta$ is the two dimensional Laplacian. We require one or the other of the boundary conditions for $e$ or $\left(h_{1}, h_{2}\right)$ :

$$
e=0 \text { or }-n_{2} h_{1}+n_{1} h_{2}=0 \text { on } \partial \Omega .
$$

We shall also insist that the internal magnetic field $\mathbf{m}$ satisfies $\mathbf{n} \times \mathbf{m}=\mathbf{0}$ on $\partial \Omega$. This condition, along with (1.5) and $(1.11)_{2}$, implies that $p$ satisfies

$$
\frac{\partial p}{\partial n}=0 \text { on } \partial \Omega
$$

Our goal is the study of long time behavior of solutions. We summarize our results below:

(i) There is an energy $\mathcal{E}\left(e, h_{1}, h_{2}, p\right)(t)$ which decreases on solutions as $t$ increases.

(ii) For the boundary condition $e=0$ on $\partial \Omega, e(x, y, t) \rightarrow 0, h_{1}(x, y, t) \rightarrow 0, h_{2}(x, y, t) \rightarrow 0$, $p(x, y, t) \rightarrow p^{\infty}(x, y)$ and $\mathcal{E}\left(e, h_{1}, h_{2}, p\right)(t) \rightarrow \mathcal{E}_{\infty}\left(p^{\infty}\right)$. Additionally, $p^{\infty}$ satisfies

$$
\delta^{2} \lambda^{2} \Delta p^{\infty}-2 \beta p^{\infty} \Phi^{\prime}\left(\left(a p^{\infty}\right)^{2}\right)=0 \text { in } \Omega \text { and } \frac{\partial p^{\infty}}{\partial n}=0 \text { on } \partial \Omega
$$

and (1.16) is the Euler equation for the critical points of $\mathcal{E}_{\infty}\left(p^{\infty}\right)$.

(iii) For the boundary condition $-n_{2} h_{1}+n_{1} h_{2}=0$ on $\partial \Omega$ conclusions (i) and (ii) continue to hold if $\sigma_{1}>0$ while if $\sigma_{1}=0, h_{1}(x, y, t) \rightarrow 0, h_{2}(x, y, t) \rightarrow 0, e(x, y, t) \rightarrow e^{\infty}$ (a constant), $p(x, y, t) \rightarrow p^{\infty}(x, y)$ and $\mathcal{E}\left(e, h_{1}, h_{2}, p\right)(t) \rightarrow \mathcal{E}_{\infty}\left(p^{\infty}\right)$. Additionally, $p^{\infty}$ satisfies

$$
\delta^{2} \lambda^{2} \Delta p^{\infty}-\beta\left(e^{\infty}-2 p^{\infty} \Phi^{\prime}\left(\left(a p^{\infty}\right)^{2}\right)\right)=0 \text { in } \Omega \text { and } \frac{\partial p^{\infty}}{\partial n}=0 \text { on } \partial \Omega
$$

and the above equation is the Euler equation for the critical points of $\mathcal{E}_{\infty}\left(p^{\infty}\right)$. The constant $e^{\infty}$ is determined by $p^{\infty}$ and the initial values of $e$ and $p$ by the formula

$$
e^{\infty}=\frac{1}{A(\Omega)} \iint_{\Omega}\left(e(x, y, 0)+p(x, y, 0)-p^{\infty}(x, y)\right) d x d y .
$$

(iv) The equilibrium problem (1.16) has multiple solutions and these will be analyzed in Section 3.

That such results are expected may be seen by looking at the spatially homogeneous version of (1.12) and (1.13). This reduced system implies that $\left(h_{1}, h_{2}\right) \equiv\left(h_{1}^{0}, h_{2}^{0}\right)$, a constant vector, and that $e$ and $p$ satisfy

$$
e_{t}+p_{t}+\sigma_{1} e=0 \text { and } \delta^{2} p_{t t}+\alpha \delta p_{t}=\beta\left(e-2 p \Phi^{\prime}\left(a^{2} p^{2}\right)\right) .
$$

If one multiplies the first equation by $\beta e$ and the second by $p_{t}$ and adds the results one obtains the identities 


$$
\frac{d}{d t}\left(\frac{\beta e^{2}}{2}+\frac{\delta^{2} p_{t}^{2}}{2}+\frac{1}{a^{2}} \Phi\left(a^{2} p^{2}\right)\right)=-\sigma_{1} e^{2}-\alpha \delta p_{t}^{2}<0
$$

and

$$
\begin{aligned}
\frac{\beta e^{2}}{2} & +\frac{\delta^{2} p_{t}^{2}}{2}+\frac{1}{a^{2}} \Phi\left(a^{2} p^{2}\right)+\int_{0}^{t}\left(\sigma_{1} e^{2}+\alpha \delta p_{s}^{2}\right)(s) d s \\
& =\frac{\beta}{2} e^{2}(0)+\frac{\delta^{2} p_{t}^{2}(0)}{2}+\frac{1}{a^{2}} \Phi\left(a^{2} p^{2}(0)\right) .
\end{aligned}
$$

If $\sigma_{1}>0, \alpha>0$, and $\delta>0$, then (1.19) - (1.21) imply that $e, p$, and $p_{t}$ are bounded and that $e$, and $p_{t}$ are in $L_{2}[0, \infty)$. Moreover, if one multiplies $(1.19)_{1}$ by $e_{t}$ and $(1.19)_{2}$ by $p_{t t}$ and notes that $\Phi^{\prime}\left(a^{2} p^{2}\right)+2 a^{2} p^{2} \Phi^{\prime \prime}\left(a^{2} p^{2}\right)$ is bounded one finds that $e_{t}$ and $p_{t t}$ are in $L_{2}[0, \infty)$ and this guarantees that $\lim _{t \rightarrow \infty} e(t)=0$ and $\lim _{t \rightarrow \infty} p_{t}(t)=0$. Finally, if one differentiates $(1.19)_{2}$ with respect to $t$ and multiplies the resulting expression by $p_{t t t}$ one finds that $p_{t t t}$ is also in $L_{2}[0, \infty)$ and this guarantees $\lim _{t \rightarrow \infty} p_{t t}(t)=0$, that $\lim _{t \rightarrow \infty} p(t) \stackrel{\text { def }}{=} p^{\infty}$ exists and that $p^{\infty}$ satisfies $2 p^{\infty} \Phi^{\prime}\left(\left(a p^{\infty}\right)^{2}\right)=0$. We are also guaranteed that the energy

$$
\mathcal{E} \stackrel{\text { def }}{=} \frac{\beta e^{2}}{2}+\frac{\delta^{2} p_{t}^{2}}{2}+\frac{1}{a^{2}} \Phi\left(a^{2} p^{2}\right)
$$

satisfies

$$
\lim _{t \rightarrow \infty} \mathcal{E}(t)=\frac{1}{a^{2}} \Phi\left(\left(a p^{\infty}\right)^{2}\right) .
$$

Our results are generalizations of these observations to spatially inhomogeneous situations.

We also note that in the limit where $\delta \rightarrow 0^{+}$the solutions of (1.12) and (1.13) exhibit microstructural oscillations where $p$ rapidly jumps from -1 to +1 and from +1 to -1 . The location of these jumps are commonly referred to as "domain-walls". Such solutions are most readily obtained in a slab geometry where $-\infty<y<\infty$ and $0<x<\ell$ and where solutions are independent of $y$.

One such solution is shown in Figures 3 and 4 below. These were run with the system (1.12) and (1.13) with the following parameter values: $\sigma_{1}=c=\lambda=\alpha=\beta=\ell=1$ and $\delta=.01$. We imposed the boundary conditions $(1.14)_{2}$ and (1.15). Our initial data was

$$
\left(h_{1}, h_{2}\right) \equiv(0,0), e \equiv 0, \text { and } p=\cos 3 \pi x, 0<x<1 \text {. }
$$

The results of this simulation are shown at time $t=50$. Our numerics conserved the zero means of $e$ and $p$.

\section{B. An Equivalent System}

We cast the system (1.12), (1.13) in a slightly more symmetric looking fashion. To effect this reduction, we assume that the initial data

$$
e^{0}(x, y)=\lim _{t \rightarrow 0^{+}} e(x, y, t),(x, y) \in \Omega
$$

and

$$
\left(h_{1}^{0}, h_{2}^{0}\right)(x, y)=\lim _{t \rightarrow 0^{+}}\left(h_{1}, h_{2}\right)(x, y, t),(x, y) \in \Omega
$$


satisfy

$$
\begin{gathered}
\frac{\partial h_{1}^{0}}{\partial x}+\frac{\partial h_{2}^{0}}{\partial y}=0,(x, y) \in \Omega \\
\left(h_{1}^{0}, h_{2}^{0}\right) \cdot\left(n_{1}, n_{2}\right)=0 \quad, \quad(x, y) \in \partial \Omega
\end{gathered}
$$

and

$$
e^{0}(x, y)=0,(x, y) \in \partial \Omega \text {. }
$$

Equations $(1.12)_{2}$ and $(1.12)_{3}$, when combined with (1.26) and (1.27) imply that

$$
\frac{\partial h_{1}}{\partial x}+\frac{\partial h_{2}}{\partial y}=0,(x, y) \in \Omega \text { and } t>0
$$

and

$$
\left(h_{1}, h_{2}\right) \cdot\left(n_{1}, n_{2}\right)=0,(x, y) \in \partial \Omega \text { and } t>0
$$

and these latter two identities imply the existence of a potential $\psi$ such that

$$
h_{1}=-c \frac{\partial \psi}{\partial y} \text { and } h_{2}=c \frac{\partial \psi}{\partial x},(x, y) \in \Omega \text { and } t \geq 0
$$

and

$$
\psi(x, y, t)=g(t),(x, y) \in \partial \Omega \text { and } t \geq 0
$$

Additionally, $(1.12)_{2}$ and $(1.12)_{3}$ imply the existence of a function $t \rightarrow H(t)$ such that

$$
e(x, y, t)=\frac{\partial \psi}{\partial t}(x, y, t)-\frac{d H}{d t}(t)
$$

for all $(x, y) \in \Omega$ and $t \geq 0$. Moreover, $(1.14)_{1},(1.32)$, and (1.33) imply that $\frac{d g}{d t}=\frac{d H}{d t}$. If we now let

$$
\phi \stackrel{\text { def }}{=} \psi-g,(x, y) \in \Omega \text { and } t \geq 0
$$

we find that

$$
e=\phi_{t} \quad, \quad h_{1}=-c \frac{\partial \phi}{\partial y} \quad, \quad \text { and } \quad h_{2}=c \frac{\partial \phi}{\partial x}
$$

and that $\phi$ satisfies

$$
\phi_{t t}+\sigma_{1} \phi_{t}+p_{t}=c^{2} \Delta \phi,(x, y) \in \Omega \text { and } t>0
$$

and

$$
\phi(x, y, t) \equiv 0,(x, y) \in \partial \Omega \text { and } t>0
$$


Moreover, $\phi$ is coupled to $p$ through

$$
\delta^{2} p_{t t}+\alpha \delta p_{t}-\beta\left(\phi_{t}-2 p \Phi^{\prime}\left(a^{2} p^{2}\right)\right)=\delta^{2} \lambda^{2} \Delta p,(x, y) \in \Omega \text { and } t>0
$$

and

$$
\frac{\partial p}{\partial n}(x, y, t)=0,(x, y) \in \partial \Omega \text { and } t>0 .
$$

In Section 2 we shall focus on (1.35) - (1.39).

\section{Large Time Behavior of Solutions to (1.35)-(1.39).}

In this section we focus on the large time behavior of solutions of the system:

$$
\begin{gathered}
\phi_{t t}+\sigma_{1} \phi_{t}+p_{t}=c^{2} \Delta \phi \quad, \quad(x, y) \in \Omega \text { and } t>0 \\
\delta^{2} p_{t t}+\alpha \delta p_{t}-\beta\left(\phi_{t}-2 p \Phi^{\prime}\left(a^{2} p^{2}\right)\right)=\delta^{2} \lambda^{2} \Delta p \quad, \quad(x, y) \in \Omega \text { and } t>0
\end{gathered}
$$

satisfying the boundary conditions

$$
\phi(x, y, t)=\frac{\partial p}{\partial n}(x, y, t)=0 \quad, \quad(x, y) \in \partial \Omega \text { and } t>0 .
$$

Once again the parameters $\alpha, \beta, \delta, \lambda$, and $c$ are positive, $\sigma_{1} \geq 0$, and $e, h_{1}$, and $h_{2}$ are related to $\phi$ by

$$
e=\phi_{t} \quad, \quad h_{1}=-c \frac{\partial \phi}{\partial y} \quad, \quad \text { and } h_{2}=c \frac{\partial \phi}{\partial x} .
$$

Information about the large time behavior of the system (2.1) - (2.3) will follow from a series of energy identities; the most basic of which is obtained by multiplying (2.1) by $\beta \phi_{t}$ and (2.2) by $p_{t}$ and adding the resulting expressions. The identity is

$$
\frac{\partial E}{\partial t}-\operatorname{divq}=-\sigma_{1} \beta \phi_{t}^{2}-\alpha \delta p_{t}^{2} \leq 0
$$

where

$$
\begin{gathered}
E=\frac{\beta}{2}\left(\phi_{t}^{2}+c^{2}|\nabla \phi|^{2}\right)+\frac{\delta^{2}}{2}\left(p_{t}^{2}+\lambda^{2}|\nabla p|^{2}\right)+\frac{\beta \Phi\left(a^{2} p^{2}\right)}{a^{2}}, \\
\mathbf{q}=\beta c^{2} \phi_{t} \nabla \phi+\delta^{2} \lambda^{2} p_{t} \nabla p,
\end{gathered}
$$

and

$$
\mathbf{q} \cdot \mathbf{n}=0 \quad, \quad(x, y) \in \partial \Omega \text { and } t>0
$$

and once again $\Phi\left(a^{2} p^{2}\right)$ is the double-well potential with a unique local maxima at $p=0$ and minima at $p \pm 1$. The key point of this and succeeding estimates is the fact that the term $\beta p_{t} \phi_{t}$ which comes from the multiplication of $(2.1)$ by $\beta \phi_{t}$ exactly cancels the term $-\beta p_{t} \phi_{t}$ which comes from multiplying (2.2) by $p_{t}$. 
To obtain the higher order estimates we differentiate the system (2.1) - (2.3) with respect to time. One differentiation implies that the pair $\left(\phi_{t}, p_{t}\right)$ satisfies

$$
\begin{gathered}
\phi_{t t t}+\sigma_{1} \phi_{t t}+p_{t t}=c^{2} \Delta \phi_{t} \quad, \quad(x, y) \in \Omega \text { and } t>0, \\
\delta^{2} p_{t t t}+\alpha \delta p_{t t}-\beta\left(\phi_{t t}-G_{1}(p) p_{t}\right)=\dot{\delta}^{2} \lambda^{2} \Delta p_{t}, \quad(x, y) \in \Omega \text { and } t>0,
\end{gathered}
$$

and the boundary conditions

$$
\phi_{t}(x, y, t)=\frac{\partial p_{t}}{\partial n}(x, y, t)=0 \quad, \quad(x, y) \in \partial \Omega \text { and } t>0,
$$

while two differentiations imply that the pair $\left(\phi_{t t}, p_{t t}\right)$ satisfies

$$
\begin{gathered}
\phi_{t t t t}+\sigma_{1} \phi_{t t t}+p_{t t t}=c^{2} \Delta \phi_{t t} \quad, \quad(x, y) \in \Omega \text { and } t>0 \\
\delta^{2} p_{t t t t}+\alpha \delta p_{t t t}-\beta\left(\phi_{t t t}-G_{1}(p) p_{t t}-G_{2}(p) p_{t}^{2}\right)=\delta^{2} \lambda^{2} \Delta p_{t t} \quad, \quad(x, y) \in \Omega \text { and } t>0
\end{gathered}
$$

and the boundary conditions

$$
\phi_{t t}(x, y, t)=\frac{\partial p_{t t}}{\partial n}(x, y, t)=0 \quad, \quad(x, y) \in \partial \Omega \text { and } t>0 .
$$

The functions $G_{1}$ and $G_{2}$ are given by

and

$$
\left.\begin{array}{l}
G_{1}(p)=2\left(\Phi^{\prime}\left(a^{2} p^{2}\right)+2 a^{2} p^{2} \Phi^{\prime \prime}\left(a^{2} p^{2}\right)\right) \\
G_{2}(p)=2 a^{2}\left(6 p \Phi^{\prime \prime}\left(a^{2} p^{2}\right)+4 a^{2} p^{3} \Phi^{\prime \prime \prime}\left(a^{2} p^{2}\right)\right)
\end{array}\right\}
$$

and they are bounded by virtue of (1.9).

Associated with the identities (2.9) - (2.11) and (2.12) - (2.14) we obtain identities of the form:

and

$$
\left.\begin{array}{rl}
\frac{\partial E}{\partial t}-\operatorname{divq}=G \quad, \quad(x, y) \in \Omega \text { and } t>0 \\
\mathbf{q} \cdot \mathbf{n}=0, \quad(x, y) \in \partial \Omega .
\end{array}\right\}
$$

Equation (2.16) implies that

$$
\frac{d}{d t} \iint_{\Omega} E(x, y, t) d x d y=\iint_{\Omega} G(x, y, t) d x d y
$$

and

$$
\iint_{\Omega} E(x, y, t) d x d y=\iint_{\Omega} E\left(x, y, 0^{+}\right) d x d y+\int_{0}^{t}\left(\iint_{\Omega} G(x, y, s) d x d y\right) d s .
$$

In the case of (2.9) - (2.11)

$$
E=\frac{\beta}{2}\left(\phi_{t t}^{2}+c^{2}\left|\nabla \phi_{t}\right|^{2}\right)+\frac{\delta^{2}}{2}\left(p_{t t}^{2}+\lambda^{2}\left|\nabla p_{t}\right|^{2}\right),
$$




$$
\mathbf{q}=\beta c^{2} \phi_{t t} \nabla \phi_{t}+\delta^{2} \lambda^{2} p_{t t} \nabla p_{t}
$$

and

$$
G=-\sigma_{1} \beta \phi_{t t}^{2}-\alpha \delta p_{t t}^{2}-\beta G_{1}(p) p_{t} p_{t t}
$$

whereas in the case of $(2.12)-(2.14)$

$$
\begin{gathered}
E=\frac{\beta}{2}\left(\phi_{t t t}^{2}+c^{2}\left|\nabla \phi_{t t}\right|^{2}\right)+\frac{\delta^{2}}{2}\left(p_{t t t}^{2}+\lambda^{2}\left|\nabla p_{t t}\right|^{2}\right), \\
\mathbf{q}=\beta c^{2}\left(\phi_{t t t} \nabla \phi_{t t}\right)+\delta^{2} \lambda^{2} p_{t t t} \nabla \phi_{t t}
\end{gathered}
$$

and

$$
G=-\sigma_{1} \beta \phi_{t t t}^{2}-\alpha \delta p_{t t t}^{2}-\beta\left(G_{1}(p) p_{t t} p_{t t t}+G_{2}(p) p_{t}^{2} p_{t t t}\right) .
$$

Next, we record some immediate consequences of the identities (2.5) and (2.15) when $E$ is given by (2.6), (2.19), and (2.22).

Lemma 1. The identities (2.5) and (2.6) imply that

(i) $\phi$ is in $H_{0}^{1}(\Omega)$ with bounds which are independent of $t$,

(ii) $p$ is in $H^{1}(\Omega)$ with bounds which are independent of $t$,

(iii) $\phi_{t}$ and $p_{t}$ are in $L_{2}(\Omega)$ with bounds which are independent of $t$,

(iv) $\alpha>0$ and $\delta>0$ imply that $p_{t}$ is in $L_{2}(\Omega \times[0, \infty))$, and

(v) if $\sigma_{1}>0$, then $\phi_{t}$ is in $L_{2}(\Omega \times[0, \infty))$.

Lemma 2. The identities (1.9), (2.15) and (2.19) and the result (iv) of Lemma 1 imply that

(i) $\phi_{t}$ is in $H_{0}^{1}(\Omega)$ with bounds which are independent of $t$,

(ii) $p_{t}$ is in $H^{1}(\Omega)$ with bounds which are independent of $t$,

(iii) $\phi_{t t}$ and $p_{t t}$ are in $L_{2}(\Omega)$ with bounds which are independent of $t$,

(iv) $\alpha>0$ and $\delta>0$ imply that $p_{t t}$ is in $L_{2}(\Omega \times[0, \infty))$,

(v) if $\sigma_{1}>0$, then $\phi_{t t}$ is in $L_{2}(\Omega \times[0, \infty))$.

Proof. The key step in establishing Lemma 2 is the observation that (1.9) implies that

$$
-\beta G_{1}(p) p_{t} p_{t t} \leq \frac{\alpha \delta}{2} p_{t t}^{2}+\frac{\beta^{2} k_{1}^{2}}{2 \alpha \delta} p_{t}^{2}
$$

and this inequality, (2.21), and the fact that Lemma 1 guarantees that $p_{t} \in L_{2}(\Omega \times[0, \infty))$ implies the results claimed. Here, $k_{1}$ is an upper bound for $\left|G_{1}(p)\right|$.

The next set of estimates will be a simple consequence of (1.9), (2.15), and (2.22) and the following inequality which pertains to functions which are $H^{1}(\Omega)$, independently of $t$, and in $L_{2}(\Omega \times[0, \infty))$, namely

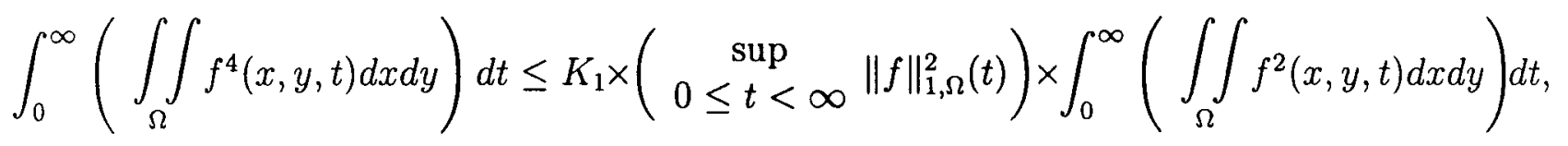

where $K_{1}$ is independent of $f$ and depends only on $\Omega^{2}$

${ }^{2}$ Here $\|f\|_{1, \Omega}^{2}(t)=\iint_{\Omega} f^{2}(x, y, t) d x d y+\iint_{\Omega}\left(f_{x}^{2}+f_{y}^{2}\right)(x, y, t) d x d y$ 
Lemma 3. The identities (1.9), (2.15), (2.22) and (2.25) imply that

(i) $\phi_{t t}$ is in $H_{0}^{1}(\Omega)$ with bounds which are independent of $t$,

(ii) $p_{t t}$ is in $H^{1}(\Omega)$ with bounds which are independent of $t$,

(iii) $\phi_{t t t}$ and $p_{t t t}$ are in $L_{2}(\Omega)$ with bounds which are independent of $t$,

(iv) $\alpha>0$ and $\delta>0$ imply that $p_{t t t}$ is in $L_{2}(\Omega \times[0, \infty))$,

(v) if $\sigma_{1}>0$, then $\phi_{t t t}$ is in $L_{2}(\Omega \times[0, \infty))$.

Proof. The key step in establishing this result is the observation that (1.9) implies that

$$
-\beta G_{1}(p) p_{t t} p_{t t t} \leq \frac{\alpha \delta}{4} p_{t t t}^{2}+\frac{\beta^{2} k_{1}^{2}}{\alpha \delta} p_{t t}^{2}
$$

and

$$
-\beta G_{2}(p) p_{t}^{2} p_{t t t} \leq \frac{\alpha \delta}{4} p_{t t t}^{2}+\frac{\beta^{2} k_{2}^{2}}{\alpha \delta} p_{t}^{4}
$$

where $k_{2}$ is an upper bound for $\left|G_{2}(p)\right|$. These inequalities guarantee that the source term $G$ defined in (2.24) satisfies

$$
G \leq-\sigma_{1} \beta \phi_{t t t}^{2}-\frac{\alpha \delta}{2} p_{t t t}^{2}+\frac{\beta^{2} k_{2}^{2}}{\alpha \delta}\left(p_{t}^{4}+p_{t t}^{2}\right)
$$

and the last inequality along with the results of Lemmas 1 and 2 and the inequality (2.25) imply the results claimed.

The underlying equations $(2.1)-(2.3),(2.9)-(2.11)$, and (2.12) - (2.14) together with the results of Lemmas 1-3 also yield

Lemma 4. (i) $\Delta \phi$ and $\Delta p$ are in $H^{1}(\Omega)$ independently of $t$ and (ii) $\Delta \phi_{t}$ and $\Delta p_{t}$ are in $L_{2}(\Omega)$ independently of $t$.

The a-priori estimates of Lemmas 1-4 imply that if the initial data is sufficiently smooth, then for each $T>0$ the functions

$$
\left(\phi^{t}, p^{t}\right)(x, y, s)=(\phi, p)(x, y, t+s) \quad, \quad(x, y) \in \Omega \quad \text { and } \quad 0 \leq s \leq T
$$

are uniformly bounded in $H^{3}(\Omega \times[0, T])$ independently of $t$ with bounds that depend on the size of the data and the number $T>0^{3}$. Moreover, the fact that $p_{t}, p_{t t}$, and $p_{t t t}$ are in $L_{2}(\Omega \times[0, \infty))$ guarantees that $p_{t}$ and $p_{t t}$ converge to zero strongly in $L_{2}(\Omega)$ as $t$ tends to infinity and additionally that the functions

$$
\left(p_{s}^{t}, p_{s s}^{t}, p_{s s s}^{t}\right)(x, y, s)=\left(p_{s}, p_{s s}, p_{s s s}\right)(x, y, t+s) \quad, \quad 0 \leq s \leq T
$$

converge to zero in $L_{2}(\Omega \times[0, T])$ as $t$ tends to infinity. Our next task is to prove

Theorem 1. For each $T>0$ the functions $\phi^{t}$ converge strongly to zero in $H^{2}(\Omega \times[0, T])$ as $t$ tends to infinity. ${ }^{4}$

\footnotetext{
${ }^{3}$ Recall that $H^{k}(\Omega \times[0, T])$ consists of all functions $f$ on $\Omega \times[0, T]$ with partial derivatives $\partial_{x}^{m} \partial_{y}^{n} \partial_{t}^{p} f$ of order $m+n+p \leq k$ which are in $L_{2}(\Omega \times[0, T])$. For such functions

$$
\|f\|_{k, T}^{2}=\sum_{m+n+p \leq k} \int_{0}^{T}\left(\iint_{\Omega}\left(\partial_{x}^{m} \partial_{y}^{n} \partial_{s}^{p} f\right)^{2}(x, y, s) d x d y\right) d s
$$

${ }^{4}$ The implications of this result for the fields $e, h_{1}$, and $h_{2}$ follow directly from (2.4). In particular, $\left(e^{t}, h^{t}, h_{2}^{t}\right)(x, y, s)=$ $\left(\phi_{s},-c \phi_{y}, c \phi_{x}\right)(x, y, t+s), 0 \leq s \leq T$ converge to zero strongly in $L_{2}(\Omega \times(0, T))$ as $t \rightarrow \infty$.
} 
Proof. We assume the theorem is false. Then, we can find an increasing sequence $\left\{t_{n}\right\}_{n=1}^{\infty}$ with $\lim _{n \rightarrow \infty} t_{n}=\infty$ and an $\epsilon>0$ such that $\left\|\phi^{t_{n}}\right\|_{2, T} \geq \epsilon$. We note that the sequence $\left\{\phi^{t_{n}}\right\}_{n=1}^{\infty}$ is also bounded in $H^{3}(\Omega \times[0, T])$ and thus we can find a subsequence $\left\{\tau_{k(n)}\right\}_{n=1}^{\infty}$ with $\lim _{n \rightarrow \infty} \tau_{k(n)}=\infty$ of the original sequence and a function $\phi^{\infty}$ in $H^{3}(\Omega \times[0, T])$ such that

$$
\lim _{n \rightarrow \infty}\left\|\phi^{\tau_{k(n)}}-\phi^{\infty}\right\|_{2, T}=0 \text { and }\left\|\phi^{\infty}\right\|_{2, T} \geq \epsilon .
$$

Moreover, we may assume, without loss of generality, that the sequence $\left\{p^{\tau_{k(n)}}\right\}_{n=1}^{\infty}$ converges strongly to $p^{\infty}$ in $H^{2}(\Omega \times[0, T])$, that $p^{\infty}$ is in $H^{3}(\Omega \times[0, T])$, and finally that $\phi^{\infty}$ and $p^{\infty}$ satisfy the limit equations

$$
\begin{gathered}
\phi_{s s}^{\infty}+\sigma_{1} \phi_{s}^{\infty}=c^{2} \Delta \phi^{\infty} \quad, \quad(x, y) \in \Omega \text { and } 0 \leq s \leq T, \\
\beta \phi_{s}^{\infty}=2 \beta p^{\infty} \Phi^{\prime}\left(\left(a p^{\infty}\right)^{2}\right)-\delta^{2} \lambda^{2} \Delta p^{\infty} \quad, \quad(x, y) \in \Omega \text { and } 0 \leq s \leq T, \\
p_{s}^{\infty}=0 \quad, \quad(x, y) \in \Omega \text { and } 0 \leq s \leq T,
\end{gathered}
$$

and

$$
\phi^{\infty}(x, y, s)=\frac{\partial p^{\infty}}{\partial n}(x, y)=0 \quad, \quad(x, y) \in \partial \Omega \text { and } 0 \leq s \leq T .
$$

If we now differentiate (2.30) with respect to $s$ and exploit (2.31) we find that $\phi_{s s}^{\infty} \equiv 0$ and this in turn reduces (2.29) to $\sigma_{1} \phi_{s}^{\infty}=c^{2} \Delta \phi^{\infty}$. If we now differentiate the last relation with respect to $s$ we find that $\Delta \phi_{s}^{\infty} \equiv 0,(x, y) \in \Omega$ and $0 \leq s \leq T$. Differentiating $(2.32)_{1}$, also yields $\phi_{s}^{\infty}=0$ on $\partial \Omega$ and these two facts in turn imply that $\phi_{s}^{\infty} \equiv 0$ for $(x, y) \in \bar{\Omega}$ and $0 \leq s \leq T$. Finally, equations (2.29) and (2.32) and the identities $\phi_{s}^{\infty} \equiv \phi_{s s}^{\infty} \equiv 0$ imply that $\phi^{\infty} \equiv 0$ in $\Omega \times[0, T]$ and this in turn yields $\left\|\phi^{\infty}\right\|_{2, T} \equiv 0$ which is a contradiction.

We note that we have made no reference in the proof as to whether $\sigma_{1}$ is positive or zero. Had we assumed $\sigma_{1}>0$, then our basic a-priori estimates would have guaranteed that $\left(\phi_{s}^{t}, \phi_{s s}^{t}, \phi_{s s s}^{t}\right)$ all converged to zero in $L_{2}(\Omega \times[0, T])$ as $t$ went to infinity and thus the limit equations (2.29) and (2.30) would have directly taken the form $\Delta \phi^{\infty} \equiv 0$ and $2 \beta p^{\infty} \Phi^{\prime}\left(\left(a p^{\infty}\right)^{2}\right)-\delta^{2} \lambda^{2} \Delta p^{\infty}=0$. These relations would then have yielded the desired result.

The preceding proof gives us considerable information about the limiting behavior of solutions of $(2.1)$ - (2.3). In particular we know that if $\left(\phi^{\infty}, p^{\infty}\right)$ is such a limit, then $\phi^{\infty} \equiv 0$ and $p^{\infty}$ is a solution of the equilibrium problem

$$
\delta^{2} \lambda^{2} \Delta p^{\infty}-2 \beta p^{\infty} \Phi^{\prime}\left(\left(a p^{\infty}\right)^{2}\right)=0 \quad, \quad(x, y) \in \Omega
$$

and

$$
\frac{\partial p^{\infty}}{\partial n}(x, y)=0 \quad, \quad(x, y) \in \partial \Omega .
$$

We further note that if $\left\{t_{n}\right\}_{n=1}^{\infty}$ is an increasing sequence of times satisfying

$$
\lim _{n \rightarrow \infty} t_{n}=\infty \text { and } \lim _{n \rightarrow \infty}\left\|p^{t_{n}}-p^{\infty}\right\|_{2, T}=0,
$$

then the energy identity (2.5) implies that the averaged energy 


$$
\mathcal{E}(t, T) \stackrel{\text { def }}{=} \int_{0}^{T}\left(\iint_{\Omega}\left(\frac{\beta}{2}\left(\phi_{s}^{2}+c^{2}|\nabla \phi|^{2}\right)+\frac{\delta^{2}}{2}\left(p_{s}^{2}+\lambda^{2}|\nabla p|^{2}\right)+\frac{\beta \Phi}{a^{2}}\left(a^{2} p^{2}\right)\right)(x, y, t+s) d x d y\right) d s / T
$$

satisfies

$$
\lim _{n \rightarrow \infty} \mathcal{E}\left(t_{n}, T\right)=\iint_{\Omega}\left(\frac{\delta^{2} \lambda^{2}}{2}\left|\nabla p^{\infty}\right|^{2}+\frac{\beta \Phi}{a^{2}}\left(a p^{\infty}\right)^{2}\right)(x, y) d x d y \stackrel{\text { def }}{=} \mathcal{E}_{\infty} ;
$$

in fact (2.5) implies that $\mathcal{E}(t, T)$ is monotone decreasing in $t$ and thus we obtain the stronger result

$$
\lim _{t \rightarrow \infty} \mathcal{E}(t, T)=\mathcal{E}_{\infty}
$$

We note that the constants $\mathcal{E}_{\infty}$ are not arbitrary, rather they must be one of the critical values of the functional

$$
E(p)=\iint_{\Omega}\left(\frac{\delta^{2} \lambda^{2}}{2}|\nabla p|^{2}+\frac{\beta \Phi}{a^{2}}\left(a^{2} p^{2}\right)\right)(x, y) d x d y
$$

as $p$ ranges over $H^{1}(\Omega)$. These critical values are the energies associated with nontrivial solutions of (2.33) and (2.34). In the next section we shall show that finding the critical points of the above functional (and the associated critical values) is equivalent to finding the critical points (and critical values) of a real valued function defined on a finite dimensional Euclidean space. The dimension of this Euclidean space is related to the size of the parameter $\frac{2 \beta\left|\Phi^{\prime}(0)\right|}{\lambda^{2} \delta^{2}}$. In the general case we have not succeeded in showing that for a fixed set of parameters and domain $\Omega$ either problem has only a finite number of critical points but we note that the results of [1] imply that in the one-dimensional case where $\Omega$ is an interval, say $(0,1)$, there are only a finite number of critical points of $E$ and thus only a finite number of critical values. We note there are always multiple solutions to (2.33) and (2.34) giving rise to a given critical value $\mathcal{E}_{\infty}$ of $E$; the evenness of $E$ implies that if $p^{\infty}$ is a nontrivial solution with energy $\mathcal{E}_{\infty}=E\left(p^{\infty}\right)$, then so is $-p^{\infty}$. The above considerations lead us to

Theorem 2. Suppose the number of pairs $(\tilde{p},-\tilde{p})$ of solutions to $(2.33)$ and (2.34) is finite and suppose further that $(\phi, p)$ is the solution to (2.1) - (2.3) corresponding to a fixed initial condition

$$
\left(\phi, \phi_{t}, p, p_{t}\right)\left(x, y, 0^{+}\right)=\left(\phi^{0}, \phi^{1}, p^{0}, p^{1}\right)(x, y),(x, y) \in \Omega
$$

which is smooth enough so that the estimates of Lemmas 1-4 obtain. Then, there exists a unique limit $\left(0, p^{*}\right)$ so that

$$
\lim _{t \rightarrow \infty}\left(\left\|\phi^{t}\right\|_{2, T}+\left\|p^{t}-p^{*}\right\|_{2, T}\right)=0 .
$$

Additionally, $p^{*}$ must be one of the solutions of (2.33) and (2.34).

Proof. The results of Theorem 1 guarantee that $\phi$ has the appropriate limiting behavior. We now assume that $p$ has no limit and we let $0,\left(p_{1},-p_{1}\right),\left(p_{2},-p_{2}\right), \quad\left(p_{M},-p_{M}\right)$ be the finite set of equilibrium solutions to (2.33) and (2.34). The hypothesis that $p$ has no limit guarantees that for each index $j=$ $0,1, \ldots, M$ we can find an $\epsilon_{j}>0$ and increasing sequence of times $t_{n}^{j}$ with $\lim _{n \rightarrow \infty} t_{n}^{j}=\infty$ such that

$$
\left\|p^{t_{n}^{j}} \mp p_{j}\right\|_{2, T} \geq \epsilon_{j}
$$


for $j=0,1 \ldots, M$ and $n=1,2, \ldots$. But the uniform boundedness of the $p^{t_{n}^{j}}$ 's in $H^{3}(\Omega \times[0, T])$ guarantees we can find an increasing subsequence $\tau_{k(n, j)}$ of the times $t_{n}^{j}$ which tends to infinity such that the function $p^{\tau_{k(n, j)}}$ converge strongly in $H^{2}(\Omega \times[0, T])$ to some solution of (2.33) and (2.34) and this contradicts $(2.41)$.

We conclude this section with some remarks about the system (1.12) and (1.13) when the boundary condition $e=0$ on $\partial \Omega$ is replaced by

$$
\left(h_{1}, h_{2}\right) \cdot\left(-n_{2}, n_{1}\right)(x, y, t)=0 \quad, \quad(x, y) \in \partial \Omega
$$

This latter condition when combined with $(1.12)_{2}$ and $(1.12)_{3}$ implies that $e$ satisfies the Neumann condition

$$
\frac{\partial e}{\partial n}(x, y, t)=0 \quad, \quad(x, y) \in \partial \Omega
$$

We again insist that $p$ satisfies (1.15). To analyze the long time behavior in this situation we could again introduce a potential $\phi$ via (1.34) and $\phi$ would again satisfy (1.36) but (1.37) would be replaced by

$$
\frac{\partial \phi}{\partial n}(x, y, t)=0 \quad, \quad(x, y) \in \partial \Omega
$$

Identical energy estimates obtain for this problem but in this situation we lose $L_{2}(\Omega)$ estimates for $\phi$ and thus cannot avail ourselves of standard compactness results to conclude that $\phi$ has the desired limiting properties as $t$ tends to infinity. Thus, when the magnetic field satisfies (2.42) and $p$ satisfies (1.15) we find it preferable to work directly with the original system (1.12) and (1.13). Here we assume that the initial data for the magnetic field, $\mathbf{h}^{\mathbf{0}}$, is divergence free and thus satisfies the compatibility condition $\int_{\Omega} \mathbf{h}^{0} \cdot \mathbf{n} d s=0$. This hypothesis guarantees that for all $t>0, \mathbf{h}$ satisfies

$$
\frac{\partial h_{1}}{\partial x}+\frac{\partial h_{2}}{\partial y}=0 \text { and } \int_{\partial \Omega} \mathbf{h} \cdot \mathbf{n} d s=0
$$

For the new boundary condition our results depend upon whether $\sigma_{1}>0$ or $\sigma_{1}=0$. When $\sigma_{1}>0, e$ and $\mathbf{h}$ converge to zero as $t$ tends to infinity and $p$ converges to a solution of (2.33) and (2.34). When $\sigma_{1}=0, e$ converges to the constant $e^{\infty}$ defined implicitly in terms of $e(\cdot, \cdot, 0), p(\cdot, \cdot, 0)$ and $p^{\infty}(\cdot, \cdot)$ by

$$
e^{\infty}=\frac{1}{\mathrm{~A}(\Omega)} \iint_{\Omega}\left(e(x, y, 0)+p(x, y, 0)-p^{\infty}(x, y)\right) d x d y
$$

where $\mathrm{A}(\Omega)$ is the area of $\Omega$ and $p$ converges to $p^{\infty}$ which now satisfies

$$
2 \beta p^{\infty} \Phi^{\prime}\left(\left(a p^{\infty}\right)^{2}\right)-\delta^{2} \lambda^{2} \Delta p^{\infty}=\beta e^{\infty}
$$

and the boundary conditions (2.34). In the case when $\sigma_{1}=0$, the magnetic field $\mathbf{h}$ also converges to zero as $t$ tends to infinity.

To establish these results we use identities satisfied by solutions of (1.12), (1.13), (1.15), and (2.42). These are obtained from our previous ones by making use of (2.4). We let

$$
E_{1}=\frac{\beta}{2}\left(e^{2}+h_{1}^{2}+h_{2}^{2}\right)+\frac{\delta^{2}}{2}\left(p_{t}^{2}+\lambda^{2}|\nabla p|^{2}\right)+\frac{\beta \Phi\left(a^{2} p^{2}\right)}{a^{2}}
$$




$$
\begin{gathered}
E_{2}=\left\{\begin{array}{c}
\frac{\beta}{2}\left(e_{t}^{2}+h_{1 t}^{2}+h_{2 t}^{2}\right)+\frac{\delta^{2}}{2}\left(p_{t t}^{2}+\lambda^{2}\left|\nabla p_{t}\right|^{2}\right) \\
\text { or equivalently } \\
\frac{\beta}{2}\left(e_{t}^{2}+c^{2}|\nabla e|^{2}\right)+\frac{\delta^{2}}{2}\left(p_{t t}^{2}+\lambda^{2}\left|\nabla p_{t}\right|^{2}\right)
\end{array}\right\} \\
E_{3}=\left\{\begin{array}{c}
\frac{\beta}{2}\left(e_{t t}^{2}+h_{1 t t}^{2}+h_{2 t t}^{2}\right)+\frac{\delta^{2}}{2}\left(p_{t t t}^{2}+\lambda^{2}\left|\nabla p_{t t}\right|^{2}\right) \\
\text { or equivalently } \\
\frac{\beta}{2}\left(e_{t t}^{2}+c^{2}\left|\nabla e_{t}\right|^{2}\right)+\frac{\delta^{2}}{2}\left(p_{t t t}^{2}+\lambda^{2}\left|\nabla p_{t t}\right|^{2}\right)
\end{array}\right\} \\
\mathbf{Q}_{1}=\beta c e\left(h_{2},-h_{1}\right)+\delta^{2} \lambda^{2} p_{t} \nabla p \\
\mathbf{Q}_{2}=\beta c e_{t}\left(h_{2 t},-h_{1 t}\right)+\delta^{2} \lambda^{2} p_{t t} \nabla p_{t}=\beta c^{2} e_{t} \nabla e+\delta^{2} \lambda^{2} p_{t t} \nabla p_{t} \\
\left(h_{2 t t,}-h_{1 t t}\right)+\delta^{2} p_{t t t} \nabla p_{t t}=\beta c^{2} e_{t t} \nabla e_{t}+\delta^{2} \lambda^{2} p_{t t t} \nabla p_{t t}, \\
\mathcal{G}_{1} \stackrel{d e f}{=}-\beta \sigma_{1} e^{2}-\alpha \delta p_{t}^{2} \\
\mathcal{G}_{2} \stackrel{\text { def }}{=}-\beta \sigma_{1} e_{t}^{2}-\alpha \delta p_{t t}^{2}-\beta G_{1}(p) p_{t} p_{t t}
\end{gathered}
$$

and

$$
\mathcal{G}_{3} \stackrel{\text { def }}{=}-\beta \sigma_{1} e_{t t}^{2}-\alpha \delta p_{t t t}^{2}-\beta\left(G_{1}(p) p_{t t}+G_{2}(p) p_{t}^{2}\right) p_{t t t}
$$

and $G_{1}$ and $G_{2}$ are defined in (2.15). It is then easily checked that for indices $i=1-3$ the following identities are satisfied by solutions of (1.12), (1.13), (1.15), and (2.42):

$$
\frac{\partial E_{i}}{\partial t}-\operatorname{div} \mathbf{Q}_{i}=\mathcal{G}_{i} \quad(x, y) \in \Omega \text { and } t>0
$$

and

$$
\mathbf{Q}_{i} \cdot \mathbf{n}(x, y, t)=0 \quad, \quad(x, y) \in \partial \Omega \text { and } t>0 .
$$

Additionally, the electric field, $e$, satisfies

$$
e_{t t}+p_{t t}=c^{2} \Delta e \quad, \quad(x, y) \in \Omega \text { and } \frac{\partial e}{\partial n}=0,(x, y) \in \partial \Omega
$$

The implications of these identities are summarized in 


\section{Lemma 5.}

(i) If the initial data for $e, \mathbf{h}$, and $p$ are sufficiently smooth, then for each $t \geq 0$ and $T>0$ the functions

$$
e^{t}(x, y, s)=e(x, y, t+s) \quad, \quad(x, y) \in \Omega \text { and } 0 \leq s \leq T
$$

and

$$
p^{t}(x, y, s)=p(x, y, t+s) \quad, \quad(x, y) \in \Omega \text { and } 0 \leq s \leq T
$$

are respectively in $H^{2}(\Omega \times[0, T])$ and $H^{3}(\Omega \times[0, T])$ with bounds which depend only on the initial data and $T$.

(ii) The derivatives $p_{t}$ and $p_{t t}$ converge strongly to zero in $L_{2}(\Omega)$ as $t \rightarrow \infty$ and $p$ also satisfies the decay estimates (2.27).

(iii) If $\sigma_{1}>0$, then $e$ and $e_{t}$ converge strongly to zero in $L_{2}(\Omega)$ as $t \rightarrow \infty$ and the functions

$$
\left(e^{t}, e_{s}^{t}, e_{s s}^{t}\right)(x, y, s)=\left(e, e_{s}, e_{s s}\right)(x, y, t+s) \quad, \quad 0 \leq s \leq T
$$

converge to zero strongly in $L_{2}(\Omega \times[0, T])$ as $t \rightarrow \infty$.

Thus, if we exploit (ii) and (iii) of the preceding lemma we find that if $(e, \mathbf{h}, p)$ is a solution of (1.12), (1.13), (1.15), and (2.42) and if $\sigma_{1}>0$, then

$$
\lim _{t \rightarrow \infty} \iint_{\Omega}\left(h_{1}^{2}+h_{2}^{2}\right)(x, y, t) d x d y=0 .
$$

To establish (2.61) note that (2.44) implies the existence of a function $\chi$ such that $h_{1}=-\chi_{y}$ and $h_{2}=\chi_{x}$. Then, (1.12) yields

$$
\Delta \chi=\omega \stackrel{\text { def }}{=} \frac{1}{c}\left(e_{t}+p_{t}\right) \quad, \quad(x, y) \in \Omega \text { and } \frac{\partial \chi}{\partial n}=0 \text { on } \partial \Omega .
$$

Moreover, the source, $\omega$, satisfies

$$
\iint_{\Omega} \omega(x, y, t) d x d y \equiv 0 \text { and } \lim _{t \rightarrow \infty} \iint_{\Omega} \omega^{2}(x, y, t) d x d y=0 .
$$

Then an expansion of $\chi$ and $\omega$ in terms of the eigenfunctions of the Laplacian on $\Omega$ with zero Neumann conditions yields the estimate

$$
\iint_{\Omega}\|\nabla \chi\|^{2} d x d y=\iint_{\Omega}\left(h_{1}^{2}+h_{2}^{2}\right)(x, y, t) d x d y \leq \frac{1}{\Lambda_{2}} \iint_{\Omega} \omega^{2}(x, y, t) d x d y,
$$

which guarantees that $\mathbf{h}$ satisfies (2.61). The constant $\wedge_{2}$ in (2.64) is the smallest positive eigenvalue of the Laplacian on $\Omega$ with eigenfunctions satisfying a zero Neumann condition on $\partial \Omega$ which are orthogonal to constants.

The situation when $\sigma_{1}=0$ is more subtle. Here we use the arguments employed earlier to show that $\phi^{\infty}$ was zero to conclude that 


$$
\lim _{t \rightarrow \infty}\left(\left\|e_{s}^{t}\right\|_{0, T}^{2}+\left\|h_{1}^{t}\right\|_{0, T}^{2}+\left\|h_{2}^{t}\right\|_{0, T}^{2}\right)=0 .
$$

Equations (2.27) and (2.65) imply that the possible limits of solutions of (1.12), (1.13), (1.15), and (2.42) consist of fields $\left(e^{\infty}, \mathbf{h}^{\infty}, p^{\infty}\right)$ where $e^{\infty}$ is a constant on $\Omega, \mathbf{h}^{\infty} \equiv 0$, and $e^{\infty}$ and $p^{\infty}$ are related by

$$
\beta e^{\infty}=2 \beta p^{\infty} \Phi^{\prime}\left(\left(a p^{\infty}\right)^{2}\right)-\delta^{2} \lambda^{2} \Delta p^{\infty} \quad, \quad(x, y) \in \Omega
$$

and

$$
\frac{\partial p^{\infty}}{\partial n}(x, y)=0 \quad, \quad(x, y) \in \partial \Omega .
$$

The constant $e^{\infty}$ is related to $p^{\infty}$ by

$$
e^{\infty}=\frac{1}{\mathrm{~A}(\Omega)} \iint_{\Omega}\left(e(x, y, 0)+p(x, y, 0)-p^{\infty}(x, y)\right) d x d y
$$

and again $\mathrm{A}(\Omega)$ is the area of $\Omega$. Finally that the averaged energy satisfies

$$
\begin{aligned}
& \lim _{t \rightarrow \infty}\left(\int_{0}^{T}\left(\iint_{\Omega} E_{1}(x, y, t+s) d x d y\right) d s\right) / T \\
& =\left(\frac{\beta}{2} \mathrm{~A}(\Omega)\left(e^{\infty}\right)^{2}+\iint_{\Omega}\left(\frac{\delta^{2} \lambda^{2}}{2}\left|\nabla p^{\infty}\right|^{2}+\frac{\beta \Phi\left(\left(a p^{\infty}\right)^{2}\right)}{a^{2}}\right)(x, y) d x d y\right)
\end{aligned}
$$

where $E_{1}$ is the energy density defined in (2.46). This concludes section 2.

\section{The Equilibrium Problem.}

In this section we examine the equilibrium problem

$$
-\Delta p+\frac{2 \beta}{\lambda^{2} \delta^{2}} p \Phi^{\prime}\left(a^{2} p^{2}\right)=0 \quad, \quad(x, y) \in \Omega
$$

and

$$
\frac{\partial p}{\partial n}=0 \quad, \quad(x, y) \in \partial \Omega .
$$

where once again $0<\delta, 0<\lambda, 0<\beta$ and $\Phi:[0, \infty) \rightarrow(-\infty, \infty)$ is smooth and satisfies

$$
\Phi(0)=0, \quad-\frac{1}{2} \leq \Phi^{\prime}(s)<0, \text { and } \Phi^{\prime \prime}(s)>0,0 \leq s ;
$$

$s=a^{2}$ is the location of the unique interior minima of $\Phi$ normalized so that $\Phi\left(a^{2}\right)<0$; and finally, as $s \rightarrow \infty$

$$
\Phi(s) \sim k\left|\Phi^{\prime}(0)\right| s, \Phi^{\prime}(s) \sim k\left|\Phi^{\prime}(0)\right|, \text { and for } p=2 \text { and } 3, \Phi^{(p)}(s)=0\left(\frac{1}{s^{p-1}}\right)
$$


for some $k>0$. In what follows we let

$$
\wedge \stackrel{\text { def }}{=} \frac{2 \beta\left|\Phi^{\prime}(0)\right|}{\lambda^{2} \delta^{2}}
$$

and note that (3.1) may be rewritten as

$$
-\Delta p+\wedge p \gamma\left(a^{2} p^{2}\right)=\wedge p
$$

where

$$
\gamma(s) \stackrel{\text { def }}{=} \frac{\Phi^{\prime}(s)-\Phi^{\prime}(0)}{\left|\Phi^{\prime}(0)\right|}
$$

satisfies

$$
\gamma(0)=0, \quad 0<\gamma^{\prime}(s), \quad \text { and } \gamma\left(a^{2}\right)=1,
$$

and the asymptotic estimates

$$
\gamma(s) \sim k+1 \text { and for } p=1 \text { and } 2, \quad \gamma^{(p)}(s)=0\left(\frac{1}{s^{p}}\right) .
$$

In what follows it will be convenient to work with the normalized potentials

$$
\hat{\Psi}(s)=\int_{0}^{s} \gamma(\eta) d \eta \text { and } \hat{\Phi}(s)=\hat{\Psi}(s)-s .
$$

The problem (3.6) and (3.2) has the trivial equilibria $p \equiv \pm 1$ and $p \equiv 0$ and the non constant equilibria $p$ satisfy the a-priori bounds $-1 \leq p \leq 1$. These inequalities follow from (3.8) and the maximum (minimum) principle for the Laplace operator.

Our basic result is that finding the non trivial equilibrium solutions of (3.6) and (3.2) is equivalent to the finite dimensional problem of finding the critical points of an even $C^{2}$ function, $\mathcal{J}$, on $R^{N}$. The integer $N$ is equal to the number of eigenvalues of $-\Delta$ (with eigenfunctions which satisfy (3.2)) which are less than $\wedge$. We assume that $\wedge_{N}<\wedge<\wedge_{N+1}$.

The function $\mathcal{J}$ has critical values satisfying

$$
\frac{\wedge \mathrm{A}(\Omega)\left(\Psi\left(a^{2}\right)-a^{2}\right)}{2 a^{2}} \leq \mathcal{J}_{\substack{\text { critical } \\ \text { value }}} \leq 0,
$$

has an isolated local maxima at $\mathbf{u}=\mathbf{0}$ satisfying $\mathcal{J}(0)=0$, and satisfies the asymptotic estimate

$$
\mathcal{J}(\mathbf{u}) \sim M\|\mathbf{u}\|^{2} \quad, \quad M>0
$$

as $\|\mathbf{u}\|$ tends to infinity. These estimates guarantee that for $\delta$ large enough

$$
\mathcal{C} \stackrel{\text { def }}{=}\left\{\mathbf{u} \in R^{N} \mid \nabla \mathbf{u} \mathcal{J}(\mathbf{u})=0\right\} \subset\left\{\mathbf{u} \in R^{N} \mid\|\mathbf{u}\|<\delta\right\} .
$$

The critical points may be obtained by examining the limit points of the gradient flow

$$
\frac{d \mathbf{u}}{d t}=-\nabla_{\mathbf{u}} \mathcal{J}(\mathbf{u})
$$


specifically, if we let

$$
\mathcal{S}(\epsilon, 0)=\left\{\mathbf{u} \in R^{N} \mid \mathcal{J}(\mathbf{u}) \equiv \epsilon\right\}
$$

and

$$
\mathcal{S}(\epsilon, \infty)=\bigcup_{\mathbf{u}_{0} \in \mathcal{S}(\epsilon, 0)}\left\{\mathbf{u}_{\infty} \in R^{N} \mid \mathbf{u}_{\infty}=\lim _{t \rightarrow \infty} \hat{\mathbf{u}}\left(t, \mathbf{u}_{0}\right) \text { where } \hat{\mathbf{u}}\left(\cdot, \mathbf{u}_{0}\right) \text { satisfies }(3.11) \text { and } \hat{\mathbf{u}}\left(0, \mathbf{u}_{0}\right)=\mathbf{u}_{0}\right\}
$$

then

$$
\mathcal{C}=\bigcup_{\epsilon \in I} \mathcal{S}(\epsilon, \infty)
$$

where $I$ is the interval $\frac{\wedge \mathrm{A}(\Omega)\left(\Psi\left(a^{2}\right)-a^{2}\right)}{2 a^{2}} \leq \epsilon \leq 0$. Though not a particularly effective computational algorithm these observations point out that solutions of (3.6) and (3.2) can be obtained by taking the limits of a finite dimensional system of differential equations rather than the infinite dimensional system described in sections 1 and 2.

Now, and in what follows, we assume $\Omega$ has a complete set of smooth eigenfunctions, $\phi_{i}$, with eigenvalues, $\wedge_{i}$, satisfying

$$
-\Delta \phi_{i}=\wedge_{i} \phi_{i} \text { in } \Omega \text { and } \frac{\partial \phi_{i}}{\partial n}=0 \text { on } \partial \Omega \text {. }
$$

The numbers $\wedge_{i}$ and $\wedge$ are ordered as indicated below

$$
0=\wedge_{1}<\wedge_{2} \leq \ldots \leq \wedge_{N}<\wedge<\wedge_{N+1} \leq \cdots \leq \wedge_{N+i}
$$

and the eigenfunctions, $\phi_{i}$, satisfy the normalization conditions

$$
\phi_{1}=\frac{1}{A^{1 / 2}(\Omega)} \text { and } \iint_{\Omega} \phi_{i} \phi_{j} d x d y=\delta_{i, j}
$$

We let

$$
\mathcal{M}_{N}=\left\{u \in H^{1}(\Omega) \mid u=\sum_{i=1}^{N} u_{i} \phi_{i}\right\}
$$

and

$$
\mathcal{N}_{N}=\left\{v \in H^{1}(\Omega) \mid v=\sum_{i=1}^{\infty} v_{i} \phi_{N+i}\right\}
$$

and note that for functions $u \in \mathcal{M}_{N}$ the $L_{2}(\Omega)$ and $H^{1}(\Omega)$ norms generate equivalent topologies whereas for functions $v \in \mathcal{N}_{N}$

$$
\|v\|_{1, \Omega}^{2} \stackrel{\text { def }}{=} \iint_{\Omega}\left(\nabla v \cdot \nabla v+v^{2}\right) d x d y=\sum_{i=1}^{\infty}\left(\wedge_{N+i}+1\right) v_{i}^{2} \leq \frac{\left(\wedge_{N+1}+1\right)}{\left(\wedge_{N+1}-\wedge\right)}\|v\|_{1, \Omega}^{2}
$$


where

$$
\|v\|\left\|_{1, \Omega}^{2} \stackrel{\text { def }}{=} \sum_{i=1}^{\infty}\left(\wedge_{N+i}-\wedge\right) v_{i}^{2} \leq\right\| v \|_{1, \Omega}^{2}
$$

Moreover, solving the equilibrium problem (3.6) and (3.2) is equivalent to finding $u \in \mathcal{M}_{N}$ and $v \in \mathcal{N}_{N}$ such that

$$
\left(\wedge_{i}-\wedge\right) u_{i}+\wedge \iint_{\Omega} \phi_{i}(u+v) \gamma\left(a^{2}(u+v)^{2}\right) d x d y=0 \quad, \quad 1 \leq i \leq N
$$

and

$$
\left(\wedge_{N+i}-\wedge\right) v_{i}+\wedge \iint_{\Omega} \phi_{N+i}(u+v) \gamma\left(a^{2}(u+v)^{2}\right) d x d y=0 \quad, \quad 1 \leq i
$$

and solving (3.22) and (3.23) is equivalent to finding the critical points $u \in \mathcal{M}_{N}$ and $v \in \mathcal{N}_{N}$ of

$$
\begin{aligned}
& J(u, v) \stackrel{\text { def }}{=} \frac{1}{2} \iint_{\Omega}\left(\nabla u \cdot \nabla u+\nabla v \cdot \nabla v-\wedge\left(u^{2}+v^{2}\right)+\frac{\wedge \Psi\left(a^{2}(u+v)^{2}\right)}{a^{2}}\right) d x d y \\
& =\frac{1}{2} \sum_{i=1}^{N}\left(\wedge_{i}-\wedge\right) u_{i}^{2}+\frac{1}{2} \sum_{i=1}^{\infty}\left(\wedge_{N+i}-\wedge\right) v_{i}^{2}+\frac{\wedge}{2} \iint_{\Omega} \frac{\Psi\left(a^{2}(u+v)^{2}\right)}{a^{2}} d x d y
\end{aligned}
$$

that is solutions of

$$
\frac{\partial J}{\partial u_{i}}=0 \quad, \quad 1 \leq i \leq N
$$

and

$$
\frac{\partial J}{\partial v_{i}}=0 \quad, \quad 1 \leq i
$$

We observe that if $u \in \mathcal{M}_{N}$ and $v \in \mathcal{N}_{N}$ satisfy (3.22) and (3.23), then the following additional identities must hold

$$
\sum_{i \in 1}^{N}\left(\wedge_{i}-\wedge\right) u_{i}^{2}+\wedge \iint_{\Omega} u(u+v) \gamma\left(a^{2}(u+v)^{2}\right) d x d y=0
$$

and

$$
\sum_{i=1}^{\infty}\left(\wedge_{N+i}-\wedge\right) v_{i}^{2}+\wedge \iint_{\Omega} v(u+v) \gamma\left(a^{2}(u+v)^{2}\right) d x d y=0
$$

These last identities imply that if $u \in \mathcal{M}_{N}$ and $v \in N_{N}$ is a critical point of $J$, then

$$
\underset{\substack{J_{\text {critical }} \text { value } \\ \text { a }}}{ }(u, v)=\frac{\wedge}{2} \iint_{\Omega}\left(\frac{\Psi\left(a^{2}(u+v)^{2}\right)}{a^{2}}-(u+v)^{2} \gamma\left(a^{2}(u+v)^{2}\right)\right) d x d y
$$


The fact the function $p \stackrel{\text { def }}{=} u+v$ satisfies (3.6) and (3.2) and the bounds $-1 \leq p \leq 1$ and the fact that $\gamma(\cdot)$ satisfies (3.8) and (3.9) guarantees that any critical value of $J$ satisfies the bounds

$$
\frac{\wedge \mathrm{A}(\Omega)}{2 a^{2}}\left(\Psi\left(a^{2}\right)-a^{2}\right) \leq J_{\substack{\text { critical } \\ \text { value }}}(u, v) \leq 0 .
$$

Moreover, the lower bound is achieved at the critical points $(u, v) \equiv( \pm 1,0)=\left( \pm A^{1 / 2}(\Omega) \phi_{1}, 0\right)$. We are interested in the other critical points of $J$.

For fixed $u \in \mathcal{M}_{N}$ we first focus on the system (3.23) (equivalently (3.26)). We note that if $v \in \mathcal{N}_{N}$ is a solution of (3.23), then (3.28) implies that

$$
\begin{aligned}
& \left(\wedge_{N+1}-\wedge\right) \iint_{\Omega} v^{2} d x d y \leq\|\| v \|_{1, \Omega}^{2} \\
& \leq \sum_{i=1}^{\infty}\left(\wedge_{N+i}-\wedge\right) v_{i}^{2}+\wedge \iint_{\Omega} \gamma\left(a^{2}(u+v)^{2}\right)\left(v^{2}\right)^{2} d x d y \\
& =-\wedge \iint_{\Omega} \gamma\left(a^{2}(u+v)^{2}\right) u v d x d y \\
& \leq \wedge(k+1)\left(\iint_{\Omega} u^{2} d x d y\right)^{1 / 2}\left(\iint_{\Omega} v^{2} d x d y\right)^{1 / 2} \\
& \leq \frac{\wedge(k+1)}{\left(\wedge_{N+1}-\wedge\right)^{\frac{1}{2}}}\left(\iint_{\Omega} u^{2} d x d y\right)^{1 / 2}\|v\|_{1, \Omega}
\end{aligned}
$$

where $k+1=\lim _{s \rightarrow \infty} \gamma(s)$ is the upper bound for $\gamma(\cdot)$ on $s \geq 0$. The Lipschitz continuity of the map $v \rightarrow T(u, v)$ together with the strong monotonicity estimate

$$
\left\|\left|v^{2}-v^{1}\right|\right\|_{1, \Omega}^{2} \leq \sum_{i=1}^{\infty}\left(v_{i}^{2}-v_{i}^{1}\right)\left(T_{i}\left(u, v^{2}\right)-T_{i}\left(u, v^{1}\right)\right)
$$

(with $T_{i}(u, v)$ defined by the expression on the left-hand side of (3.23)) guarantees that for each $u \in \mathcal{M}_{N}$ there is a unique $v=\hat{v}(u) \in \mathcal{N}_{N}$ satisfying (3.23). Moreover, (3.4) - (3.9) imply that this mapping is $C^{2}$ on $\mathcal{M}_{N}$ and has the following additional properties:

$$
\begin{gathered}
\hat{v}(-u)=-\hat{v}(u), \\
\hat{v}_{i}(t e)=\frac{-\wedge \gamma^{(1)}(0) t^{3} a^{2}}{\wedge_{N+i}-\wedge} \iint_{\Omega} \phi_{N+i} e^{3} d x d y \quad, \quad \text { as } t \rightarrow 0^{+},
\end{gathered}
$$

and

$$
\hat{v}(t e) / t=o(1) \quad, \quad \text { as } t \rightarrow \infty
$$


In (3.33) and (3.34), $e=\sum_{i \in 1}^{N} e_{i} \phi_{i}$ and $\iint_{\Omega} e^{2} d x d y=\sum_{i=1}^{N} e_{i}^{2}=1$. To obtain the smoothness of the map $u \in \mathcal{M}_{N} \rightarrow v \in \mathcal{N}_{N}$ one formally differentiates (3.23) with respect to the parameters $u_{p}$ where $u=\sum_{p=1}^{N} u_{p} \phi_{p}$ to obtain integral equations for the partial derivatives $\frac{\partial v_{i}}{\partial u_{p}}, \frac{\partial^{2} v_{i}}{\partial u_{p} \partial u_{q}}$, and $\frac{\partial^{3} v_{i}}{\partial u_{p} \partial u_{q} \partial u_{r}}$. The integral operators involved are positive definite and one obtains a-priori estimates similar to (3.31) which imply the result claimed.

We now turn our attention to the system (3.22) where

$$
v=\hat{v}(u) \stackrel{\text { def }}{=} \sum_{i=1}^{\infty} \hat{v}_{i}(u) \phi_{N+i}
$$

and the $\hat{v}_{i}(u)$ 's are the unique solution of (3.23). Once again the solutions $u=\sum_{i=1}^{N} u_{i} \phi_{i}$ of this system are critical points of

$$
J(u, \hat{v}(u))=\frac{1}{2} \sum_{i=1}^{N}\left(\wedge_{i}-\wedge\right) u_{i}^{2}+\frac{1}{2} \sum_{i=1}^{\infty}\left(\wedge_{N+i}-\wedge\right) \hat{v}_{i}^{2}(u)+\frac{\wedge}{2 a^{2}} \iint_{\Omega} \Psi\left(a^{2}(u+\hat{v}(u))^{2}\right) d x d y
$$

that is the solutions of $\frac{\partial J}{\partial u_{i}}=0, i=1,2, \ldots N$. Moreover, the fact that $v=\hat{v}(u)$ satisfies (3.28) implies that $J(u, \hat{v}(u))$ reduces to

$\mathcal{J}(\mathbf{u}) \stackrel{\text { def }}{=} J(u, \hat{v}(u))=\frac{1}{2} \sum_{i=1}^{N}\left(\wedge_{i}-\wedge\right) u_{i}^{2}+\frac{\wedge}{2} \iint_{\Omega}\left(\frac{\Psi\left(a^{2}(u+\hat{v}(u))^{2}\right)}{a^{2}}-\hat{v}(u)(u+\hat{v}(u)) \gamma\left(a^{2}(u+\hat{v}(u))^{2}\right)\right) d x d y$

and the inequality (3.30) implies that critical values of $\mathcal{J}(\cdot)$ also satisfy $(3.30)$.

We now record some facts about $\mathcal{J}(\cdot)$. The first is that

$$
\frac{\partial \mathcal{J}}{\partial u_{i}}(\mathbf{u})=\frac{\partial J}{\partial u_{i}}(u, \hat{v}(u)) \quad, \quad 1 \leq i \leq N
$$

This identity follows from the fact that $v=\hat{v}(u)$ satisfies (3.26). An immediate consequence of (3.37) is the identity

$$
\begin{aligned}
\frac{\partial^{2} \mathcal{J}}{\partial u_{i} \partial u_{j}} & =\left(\wedge_{i}-\wedge\right) \delta_{i j}+\iint_{\Omega} \phi_{i} \phi_{j}\left(\gamma\left(a^{2}(u+\hat{v}(u))^{2}\right)+2\left(a^{2}(u+\hat{v}(u))^{2} \gamma^{(1)}\left(a^{2}(u+\hat{v}(u))^{2}\right)\right) d x d y\right. \\
& +\wedge \iint_{\Omega} \phi_{i}\left(\gamma\left(a^{2}(u+\hat{v}(u))^{2}\right)+2 a^{2}(u+\hat{v}(u))^{2} \gamma^{(1)}\left(a^{2}(u+\hat{v}(u))^{2}\right) \sum_{p=1}^{\infty} \frac{\partial \hat{v}_{p}}{\partial u_{j}}(u) \phi_{p}\right) d x d y
\end{aligned}
$$

The asymptotic estimate (3.33) guarantees that $\hat{v}(0)=0$ and $\frac{\partial \hat{v}}{\partial u_{j}}(0)=0$ and these identities, along with (3.9) and (3.22), imply that 


$$
\frac{\partial \mathcal{J}}{\partial u_{i}}(0)=0 \text { and } \frac{\partial^{2} \mathcal{J}}{\partial u_{i} \partial u_{j}}(0)=\operatorname{diag}\left(\wedge_{1}-\wedge, \wedge_{2}-\wedge, \ldots, \wedge_{N}-\wedge\right)
$$

and thus that $\mathbf{u}=0$ is an isolated local maxima of $\mathcal{J}$. We note that $\mathcal{J}(\mathbf{0})=0$. The asymptotic estimates (3.9) and (3.36) guarantee that for $e$ 's satisfying

$$
e=\sum_{i=1}^{N} e_{i} \phi_{i} \text { and } \iint_{\Omega} e^{2} d x d y=\sum_{i=1}^{N} e_{i}^{2}=1
$$

$\mathcal{J}$ satisfies

$$
\mathcal{J}(t \mathbf{e}) \sim \frac{t^{2}}{2}\left(\sum_{i=1}^{N} \wedge_{i} e_{i}^{2}+\wedge k\right) \text { as } t \rightarrow \infty
$$

and the latter estimate, together with the fact that the critical values of $\mathcal{J}$ satisfy (3.30), guarantees that for $\delta$ large enough all critical points of $\mathcal{J}$ satisfy (3.10) - (3.14).

We can also apply the Lyusternik-Schnirelman theory (see e.g. [4], [5]) to the function $\mathcal{J}(\mathbf{u})$ on $\mathcal{M}_{N}$ to determine the critical levels of this functional and corresponding non-trivial solutions of

$$
\nabla_{\mathbf{u}} \mathcal{J}(\mathbf{u})=0
$$

With the exception of the constant solutions, these can be expected to be saddles rather than local maxima or minima. These critical values may be obtained as follows. Let $\sum_{n}, n=1,2, \ldots, N$ denote the collection of compact, balanced (i.e. invariant under the map $\mathbf{u} \rightarrow-\mathbf{u}$ ) subsets $S \subseteq \mathcal{M}_{N} \backslash\{0\}$ of genus $\leq n$. The genus of a compact, balanced subset of $\mathcal{M}_{N} \backslash\{0\}$ is the least integer $n$ such that there exists an odd map $f: S \rightarrow \mathrm{S}^{n-1}$ ( the $(n-1)$-sphere); clearly for $S$ as above the genus is less than or equal to $N$ and by the Borsuk-Ulam theorem an $n$-sphere has genus $n+1$; for more details see [4], [5].

If we let

$$
c_{n}=\min _{S \in \sum_{n}} \max _{\mathbf{u} \in S} \mathcal{J}(\mathbf{u}), \quad n=1, \ldots N
$$

then

$$
\frac{\wedge A(\Omega)\left(\Psi\left(a^{2}\right)-a^{2}\right)}{2 a^{2}}=c_{1} \leq c_{2} \leq \ldots \leq c_{N}<0
$$

The left-most identity follows from (3.30), the monotonicity of the $c_{n}$ 's from the definition (3.43), and the last inequality from the fact that $\mathbf{u}=0$ is an isolated local maximum. It can also be shown that $c_{1}<c_{2}$.

By a standard application of the Lyusternik-Schirelman theory it follows that the $c_{n}$ 's defined by (3.43) are critical values of $\mathcal{J}(\mathbf{u})$. If these numbers are distinct, this implies the existence of at least $N$ pairs of solutions to (3.42). If there is repetition, i.e. if

$$
c_{j}=c_{j+1}=\ldots=c_{j+k-1}
$$

for some $j: 1 \leq j \leq N-k+1$ then the set of solutions to (3.42) on the level $c_{j}$ is a set of genus $k$. In particular a set of solutions of genus $k$ will contain $k$ pairs $\left(\mathbf{u}_{i},-\mathbf{u}_{i}\right), i=1, \ldots, k$ with inner product $\mathbf{u}_{i} \cdot \mathbf{u}_{j}=\delta_{i j}$.

This concludes section 3 and the paper. 


\section{References}

[1] J.M. Greenberg, "Elastic Phase Transitions-A New Model", Physica D , 108, 209-235 (1997).

[2] L. D. Landau and E. M. Lifshitz, "Electrodynamics of Continuous Media", Pergamon Press, Oxford, 1960.

[3] R.D. James and D. Kinderlehrer, "Frustration in ferromagnetic materials", Continuum Mechanics and Thermodynamics 2, 215-239 (1990).

[4] S.N. Chow \& J. Hale, "Methods of bifurcation theory", Springer Verlag, New York, 1982.

[5] P. Rabinowitz, "Some aspects of nonlinear eigenvalue problems", Rocky Mountain J., 3 161-202. 